\title{
Reflecting on Exchanged Students' Learning: Bedside Demonstration, Case Reporting and Clinical Reasoning
}

Dan Xu ( $\nabla$ daniel.xu@curtin.edu.au )

Curtin University

\section{Shaoting Feng}

First Affiliated Hospital of Sun Yat-sen University

\section{Suqing Yang}

First Affiliated Hospital of Sun Yat-sen University

\section{Shuqing Ding}

First Affiliated Hospital of Sun Yat-sen University

\section{Timothy Yap}

Curtin University

Christopher Chi

Curtin University

Nicole Tan

Curtin University

\section{Ankith Nair}

Curtin University

Dennis Nixon

Curtin University

\section{Cleo Wee}

Curtin University

\section{Johan Rosman}

Curtin University

\section{Sally Sandover}

Curtin University

\section{Jingsong Wang}

First Affiliated Hospital of Sun Yat-sen University

Ming Kuang

First Affiliated Hospital of Sun Yat-sen University

\section{Haipeng Xiao}

First Affiliated Hospital of Sun Yat-sen University 


\section{Research Article}

Keywords: clinical reasoning skills, First Affiliated hospital, academic performance, professionalism Posted Date: April 30th, 2021

DOI: https://doi.org/10.21203/rs.3.rs-461202/v1

License: (c) (i) This work is licensed under a Creative Commons Attribution 4.0 International License. Read Full License 


\section{Abstract}

Background: The increasing opportunities for medical students to participate in international, supervised and structured electives have been shown in our recent study, which enhanced students' professionalism, cultural competence and clinical reasoning skills in preclinical years. However, evidence is scant in achieving the specific learning objectives and improving clinical reasoning during clinical years. This study aims to demonstrate how the short-term supervised elective can improve students' clinical reasoning skills, skills of case report writing and satisfactory academic performance.

Results: We recorded daily discussions on the demonstrated skills and cases collection with six Curtin Medical School students from Australia during a 19-day supervised elective at the First Affiliated hospital, Sun Yat-sen University, China. The daily clinical reasoning discussions, case collection and bedside demonstrations in different disciplines became the main content of the reflections discussed in this article. Their feedback was recorded post-elective to examine the impact of the exchange in their ongoing case report writing, clinical reasoning skills development and academic performance. The main themes of case-based clinical reasoning identified in the daily discussions and feedbacks were described by the students in two frameworks. Learning through clinical reasoning is well demonstrated during studentssupervisors daily debrief. The students have used these clinical reasoning skills in their ensuing clinical placement with satisfactory academic performances. The students have completed two case reports individually with cases for publication in both Australian Doctor and BMJ case report. The supervisors obtained students' feedbacks at the time of the elective, 6-month and 12-month post-elective, and feedback summary is highlighted in three aspects including improved clinical reasoning skills, scientific writing skills and satisfactory academic performance.

Conclusions: These reflections provide insight into how this exchange elective may improve students' clinical reasoning, enhance scientific writing skills and strengthen academic performance.

\section{Introduction}

The increasing opportunities for medical students to participate in international, supervised, and structured electives have been shown in recent study ${ }^{1}$ enhancing students' professionalism, cultural competence, and clinical reasoning introduction in preclinical-years. However, evidence is scant in achieving the same goals on clinical reasoning during clinical-years. This study aims to demonstrate how short-term supervised elective can improve students' clinical reasoning skills to empower ongoing selfdirected clinical learning through bedside demonstration, case presentation, case report writing for publication. The integration of bedside demonstration and case report writing into clinical reasoning is the central theme for this discussion.

\section{Methods}

\section{Setting}


Curtin Medical School is a new medical school in Perth, Australia. The six students from Curtin Medical School were in their transition year from preclinical year to the combined preclinical-clinical and fullclinical year. The First Affiliated Hospital, Sun Yat-sen University, Guangzhou, China is one of China's most prestigious hospitals, hosting medical students enrolled in the Sun Yat-sen Medical School. The hospital is ranked second in terms of undergraduate teaching and fifth in clinical services and medical research in China. Curtin Medical School and Sun Yat-sen Medical School jointly developed the Specific Learning Objectives for the students prior to the commencement of the program. The 19-day program included a 14-day academic program at the First Affiliated Hospital, Macau hospital and Sun Yat-sen University Eye Hospital. The fourteen-day academic program was pre-planned and the students would visit one or two disciplines daily, based on the outlined Anticipated Learning Opportunities and Case Selection. Table 1 illustrated the anticipated learning opportunities and case selection and academic schedules.

\section{Participants and analysis}

During the nineteen-day period, six Curtin Medical School students attended the academic schedules listed in Table 1 under joint supervision. During the daily ward rounds, students were exposed to case studies in medicine, surgery, pathology, laboratory medicine and radiology. The daily schedule included the morning and afternoon sessions with either the end-of-session group and individual debrief or the end-of-the-day summary. The key takeaways from both individual and group debriefs form the reflections in this article. Both individual and group debriefs were conducted using both the unstructured and semistructured individual interview format, with the unstructured format being the most predominant. A semistructured individual interview format is the most widely used method in qualitative research, and enables students to delve deeply and reflect on their experiences in anticipation of the emergence of new themes during the reflection. ${ }^{2}$ The design of individual interviews is based on the modified SNAPPS 6-step feedback tool including the following sets of questions: 'have you Summarised briefly the history and examination problem lists?', 'have you Narrowed the problem lists to the two commonest possibilities and one "not-to-missed" red-flag possibility?', 'have you Analysed the problem lists by comparing and contrasting the possibilities?', 'have you Probed the supervisor by asking questions about uncertainties, difficulties, or alternative approaches?', 'have you Picked the unique case and plan for the case writing for journal submission?', and 'have you Selected learning points for both case reporting and ongoing selfdirected learning in the topic?'3 All the reflections were recorded immediately after debrief by the supervisor, then read and analysed by the thematic synthesis method including the development of descriptive themes (SNAPPS in this article) and eventual generation of analytical themes (reporting cases and learning points in this article). ${ }^{4}$ We chose this approach because it is suitable for analysing relatively unstructured, text-based data in an inclusive and rigorous manner. ${ }^{5}$ The students read the transcripts and discussed with supervisors the identified cases for case report writing and case-specific clinical-reasoning learning. This dual process was adopted to achieve higher reliability of the reflection, consequently strengthening the learning of both case reporting and clinical reasoning. Some of the case presentations and bedside demonstrations were selected for journal submission. The students have also provided 6- 
months post-elective feedback regarding the impact on clinical reasoning learning as well as academic outcomes.

\section{Results}

The students' reflections on the elective's impact on their bedside assessment skills, case report writing, clinical reasoning and academic performance have formed the results of this article. During bedside demonstration, students mastered the modified SNAPPS framework as an important clinical assessment tool (Table 2). During case presentations, 123 cases in 23 disciplines were distributed amongst the six students with each student having an average of 20 case presentations during the elective. Each student selected two cases to write case report for journal submission by using PICO framework (Table 2). Clinical reasoning has been constantly taught to the students by bedside case presentation and case report writing for submissions to Australian Doctor and British Medical Journal (BMJ) Case Report, and observed improvement has been well reflected in students' clinical placement, case report writing and academic performance.

Student one: I found my supervised elective rewarding. The bedside demonstration provided ample opportunities for observing how senior clinicians exercised "SNAPP/PICO" to obtain a comprehensive management plan. My bedside clinical assessment has been unique, as I was allowed unprecedented access to patients' entire clinical journeys, from admission to discharge to practice "SNAPP/PICO".

The process of selecting case presentation/report writing helped me to understand the complexity and the importance of clinical reasoning in prioritising management, refine my history-taking skills, understand clinical sequelae and gain confidence in patient-centred communication. I can foresee the "SNAPPS" and "PICO" frameworks being effective clinical reasoning tools for future curricular study, internship and career medical practice.

Six months onwards, case report writing created a platform for the academic-nurturing interaction between supervisors and students. These ongoing interactions through case report writing and editing have strengthened my clinical reasoning skills in my clinical placements in rural Perth, in which I have been appraised by supervisors. My Objective Structured Clinical Examination (OSCE) skill has improved through case report writing in time for my exam preparation achieving satisfactory academic results. I am now confident in clinical assessment during my clinical rotations, which will help my intern year in 2022 and serve me well to become a competent clinician.

Student two: Our exchange-elective to China was incredibly valuable in developing our approach to clinical reasoning. Interacting with patients under supervision allowed us to understand the clinical reasoning process to reach a diagnosis. Bedside clinical assessment allowed using the "SNAPPS/PICO" frameworks for selecting two case; a case of External Counterpulsation, a device used to increase blood return to coronary vessels during diastole, and a case of relapsing polychondritis, a disease diagnosed by exclusion. Case reporting writing was effective in improving my clinical approach in comprehensive history-taking, examination, and relevant investigations, also showed me the necessity of explorative 
process to avoid missing a red-flag diagnosis. Ultimately, the experience has highlighted diagnostic clinical reasoning in enquiring broadly without confining to a diagnosis early, while critical thinking with systematic approach has put me ahead in my transition into full-clinical years.

Now 6-months on, I have commenced clinical rotations at rural hospital in Australia, and observed the difference in types of cases presenting to a tertiary hospital in China in comparison to Australia. I found the China elective extremely valuable in my clinical reasoning skills transition into full-time placement application through exposing me to large volumes of clinical cases in different body-systems at clinical setting. During these placements, I have used "SNAPPS" and "PICO" frameworks in my daily clinical encounters and was constantly watching for case report writing to share with fellow students and supervisors, whom were impressed with my presentation of the case I saw during the elective.

Student three: "SNAPPS/PICO" was a great outline to keep in my mind entering the exchange program second-time, this goal-orientated approach allowed me to clinical reasoning skills to obtain key information required to complete the case reports. Information gathering through this approach was invaluable both clinically and professionally for education and collegiality. Under close supervision, I completed two case reports, a case of near-fatal Vibrio Vulnificus Infection leading to amputation, a case of carotid artery dissection with massive stroke caused by chiropractic massage. Daily reflection with SNAPPS/PICO on the cases and recalling the bedside interaction was an effective way to consolidate clinical reasoning learning from experienced clinicians. Case report writing is an important skill to allow clinicians sharing and disseminating knowledge especially, learning from mistakes, guiding evidencebased practice. As medical students with limited experience and opportunities, case report writing provided the stepping stone to develop both clinical assessment and reasoning skills, thus decision making. This experience has facilitated better transition into full-time case-based learning and effectively retaining critical information. Ultimately, the case report writing and clinical reasoning skills through this elective was vital to my progression from learning to critical thinking.

The last six months of clinical rotations have highlighted the value of the elective with efficient use of SNAPPS/PICO daily to consolidate knowledge, promote confidence and critical thinking. The rare cases encountered were also invaluable as I was able to share my experience with clinicians and get an alternate perspective or experience from their careers, motivating ongoing study. Finally, the case report writing has refined my clinical writing skills of getting salient information, enhancing clinical reasoning skills, thus contributing to OSCE and competent intern preparation.

Student four: By applying the "SNAPPS/PICO" framework, the elective enabled us to improve our clinical reasoning in an engaging manner. After taking histories and performing examinations on patients, we brainstormed and excluded possible differentials as a team. A salient learning point was the process of systematically excluding differentials by analysing the patient's history, physical examination and investigation results. Getting involved with case reporting has provided incredibly valuable insight into the thinking processes medical students should adopt when approaching patients. This process was well reflected by two selected case reports. One was a case of recurrent pneumonia caused by a 
tracheoesophageal fistula and the other discussed acute retinal necrosis in an immunocompetent patient. Writing the case reports has reinforced the use of SNAPPS/PICO when approaching patients. This skill will assist me in my clinical years and postgraduate training.

On reflection six months later, I have found the SNAPPS framework useful in conjugation with our PBL (problem-based learning) cases. This become particularly useful as contact hours at university were reduced due to the COVID-19 pandemic, and having a systematic approach to new cases enabled me to further self-direct my own learning.

Student five: An intensive two-weeks elective to practise clinical assessment skills daily with bedside demonstration by using the "SNAPPS/PICO" framework has changed the way I study medicine for the better. Supervised history taking highlighted the importance of having a systematic and logical approach, categorising differentials in an organised manner either by body-systems, pathological processes, or by 'can't be missed' - red flag diagnoses. This approach has become the centre of my studies after the elective, allowing me to envisage theory into a clinical context. As a result, I now write down three-to-five pertinent questions to ask for every disease/pathology, making me to choose wisely on relevant investigations and management. I prepared two case reports including one case on achalasia in a patient with progressively worsening dysphagia and another case about cervical spine abscess and osteomyelitis in a patient with rapidly progressive paralysis. In preparing both case reports, I was using the SNAPPS/PICO by taking 'clues' that can assist in narrowing down the diagnosis and establishing the most appropriate management. I found the task to write case reports after the bed side history taking essential in reinforcing the knowledge I learnt in wards and teaching me the real-life clinical reasoning process, which I needs to become a competent intern as well as preparing my OSCE.

My ongoing study six months after the elective has greatly reminded me the "SNAPPS/PICO" framework used for clinical assessment and case selection. The COVID-19 pandemic has had a significant impact on our contact hours at university, and studying medicine became more self-directed than ever before. I really learnt to appreciate the bedside demonstration, case presentations and clinical reasoning during the elective, through simulated patients I found "SNAPPS/PICO" framework can prioritise my clinical reasoning learning to progress me to be a competent intern.

Student six: The elective has given the opportunity to develop our clinical reasoning skills, primarily through "SNAPPS/PICO" during bedside demonstrations and case report writing. "SNAPPS/PICO" allowed me to identify the key points in a given case with more clarity and direction. The demonstration of clinical skills in a structured bedside setting helped shed light on the thought process clinicians undergo. The active participation in clinical reasoning with experienced doctors has built the confidence of patient interaction, facilitating case identification and introducing case report writing. Writing case report allowed me to dissect and reflect on the clinical reasoning process for a final diagnosis under the close supervision of experienced doctors, whom discussed the case with us in great detail, allowing us to develop a deep understanding and appreciation of the clinical reasoning process. 
Six-month on, the elective has paid great dividends in three areas including knowledge retention, clinical reasoning and scientific writing. A wide range of patients with various conditions, both common and rare, enhanced my knowledge retention by making the link of pre-clinical learning with real patients. The various rare cases extended the learning boundary to case report writing. Scientific writing is fundamental for career clinicians, introduction of case report writing included one patient with aortic dissection and another with sciatic nerve endometriosis. The case report writing built starting points for scientific writing and research. Clinical reasoning learning has been efficacious through bedside clinical assessment using "SNAPPS/PICO" and interacting with editors and supervisors when answering editorial questions, a boost to OSCE preparation.

\section{Discussion}

This reflection has demonstrated how clinical reasoning was taught and learned through bedside demonstration, case presentation with reflected discussion, and case selection for case report writing. The ultimate goals of this reflection will include improving students' clinical competency, reducing theorypractice gap, consolidating the learning of clinical reasoning, and understanding the essential preparations to become a qualified intern.

\section{Bedside clinical skill demonstration}

Bedside clinical teaching is known to be difficult to implement because it is obviously resource intensive with many factors including increasing workload and need for efficiency in clinical departments, service provision, and patient care, which may take priority over teaching when resources are limited. ${ }^{6}$ There may be factors specific to the learner, teacher, patient, environment, and ethical issues. However, bedside teaching has been well documented to provide benefits in terms of role modelling, staff recruitment and retention, formative assessment of learner's performance, continuing professional development of the teacher and quality improvement, both clinically and educationally. The elective students had been provided with formal bedside teaching including pre-selected cases, two dedicated tutors, and inpatient availability of all departments. Recent evidence has demonstrated that formal bedside teaching is effective if organised with adequate staffing to quarantine the teachers or tutors, and concentrating on case presentation, case selection and clinical reasoning discussion. ${ }^{6}$ Clinical reasoning and knowledge were perceived to be most important, exerting good patient outcome through comprehensive assessment with "SNAPPS" and "PICO" frameworks leading to prompt decision making. Bedside demonstration has laid the foundation for the eventual case presentation and selection for writing case report and integrate the clinical reasoning into the learning objectives. Bedside demonstrations have also been shown to be useful for providing an overview of the complete set of clinical skills to be learned, especially when an overview is provided early in the learning process of preclinical and transitional clinical years, as evident in this elective of how clinical reasoning skills were taught and learned. ${ }^{7,8,9}$

Another salient point from the reflection is case presentation and selection for case report writing for journal submission. It has been proven to be an expected outcome of this elective in enhancing clinical 
reasoning skill. Case presentation has always been one of the most valuable and evidence-based tools of medical education for presenting challenging medical cases to medical students, junior doctors and even consultant physicians. ${ }^{10}$ Clinical learning during medical school is mainly case-based. ${ }^{11}$ Most of the important educational objectives that case reports introduce include enhancing awareness of rare disorders to facilitate diagnosis, clarifying new aspects on disease's aetiology, clarifying misunderstood treatment response, and describing how to avoid future mistakes. ${ }^{12}$ During this elective, students have been able to select the cases from their many bedside demonstrations and recognize the answerable clinical question, and then to find current best evidence to answer this question by performing a thorough and effective literature review. During the literature review, students critically analysed the medical literature and chose the appropriate reference to support the case. By writing a case report, students gain experience in literature review and medical writing as well as experiencing the steps of evidence-based medicine, which consists of formulating a clinical question, finding the best evidence, critically appraising the evidence, and applying the evidence to the patient. Each of the six students has already had one case report published/accepted for publication in Australian Doctor, which is the most read clinical journals among clinicians. Another case for each student is either published or editorial review in BMJ Case Report. Even if the manuscript does not ultimately get published, the review of the case literature carries educational value. A case report including evaluating a patient's medical history, performing a physical examination, considering various differential diagnosis, selecting a treatment plan, and considering various side effects and outcomes of treatments provide an educational platform for students' clinical reasoning learning, albeit will not have as much potential impact on clinical practice as randomized controlled trials or other research. ${ }^{13}$ However, well-written and appropriately structured case reports with meticulous attention to the very minute details will contribute to the medical literature and can still enrich our knowledge in today's evidence-based medical education. The case reports written by the students from this elective certainly reflect this important educational message despite the fact that they are inexperienced and novice authors.

In line with the students' reflection, case reports provide the opportunity to engage in simpler scientific writing before pursuing more advanced forms of medical writing. Case reports provide an early opportunity to publish outside formal scientific research projects. ${ }^{14,15}$ They engage a pertinent clinical question, and give students practice in research and assessment skills that forge competent clinicians. ${ }^{16}$ Another recent study identified five educational benefits of case reports for medical students including developing observation and pattern recognition skills, developing hypothesis-generating skills, understanding patient-centered care, writing skills and rhetorical versatility, and the case report as a "minithesis". ${ }^{17}$ Such benefits have been observed in the clinical placement after the elective, when students learn self-criticism, hone hypothesis-generating skills through case report writing and answer editors' arguments and criticisms by analysing how to implement the most up-to-date research evidence into clinical practice. Implementing updated research evidence into clinical practice is the fundamental element of ongoing training for a competent clinician, as evidenced by a recent study. ${ }^{18}$ In this study, graduate trainees stated that they acquired most of their competences of implementing research into practice by on-the-job experience of clinical case management, self-study and ongoing professional 
education. Case reporting writing with this elective has certainly laid the foundation of ongoing training for medical students in terms of evidence-implementation skills in clinical practice, consequently fostering the lifelong learning of a competent clinician.

This elective highlighted two other educational points including students' longitudinal follow-up reflections at different time-points and the integration of bedside demonstration and case report writing into clinical reasoning. Clinical reasoning has been defined as a way of critical thinking and decision making in clinical practice ${ }^{19}$, requiring clinicians to analyse a cluster of clinical presentations, create a list of differential diagnosis and develop a management strategy. ${ }^{20}$ The process of clinical reasoning is undertaken by all clinicians, usually automatically, similar to the "SNAPPS" and "PICO" approaches used in this elective, and is the cognitive process that underlies differential diagnosis and management of clinical presentations. ${ }^{21}$ The dual cognitive process from cognitive psychology theory $22,23,24$ showed that the reasoning process consists of System 1 and System 2 processes. ${ }^{25,26}$ System 1 is an intuitive thinking process, which is unconscious and quick but at the same time it is prone to various cognitive biases $^{27}$, while System 2 is an analytical thinking process, which is deliberate and slow ${ }^{28}$ to reduce System 1 biases physicians use instead with System 2 process that assesses whether a diagnosis made using the intuitive process is correct or not by analysing more information. ${ }^{29,30}$ The combination of System 1 and 2 will certainly ensure the safest outcome of the clinical reasoning process applied by the clinician. Recent research indicated clinical reasoning is fundamental to medical education and practice $^{31}$ and was one of the most important indicators of competent clinicians. However, clinical reasoning is often regarded as difficult to conceptualise and teach, posing challenges to clinical teachers. ${ }^{32,33}$ Recent evidence showed motivated clinicians showcasing the pivotal role of clinical reasoning for more efficient teaching and practice in systematic and evidence-based manner, making clinical reasoning being regarded as an art rather than a science. ${ }^{34}$ This reflection paper has certainly focussed on promoting the above-mentioned medical educational points of clinical reasoning through the students' reflections during the elective and six months after. CR learned by the students during this elective and after is using system 2 by dissecting the clinical management steps through case report writing. Selecting an appropriate case to report for publication is how system 2 was used in practice. The academic benefits of writing a case report for journal submission will inform curricular development in terms of theoretical study, OSCE exam and clinical placement.

CR learned by the students through bedside demonstration and case report writing during this elective has a similar approach as the Script Concordance Test (SCT) being increasingly used in ongoing postgraduate medical education in CR. Script theory explains how physicians progressively acquire knowledge adapted to their clinical tasks. ${ }^{35,36}$ The SCT is a tool for assessment of clinical reasoning that is increasingly being used in continuing professional development in medical education. ${ }^{37}$ SCT is the unique form of clinical assessment based on clinical scenarios designed to measure clinical data interpretation. An expert reference panel, including 10-20 members with different disciplines, are recommended for optimal reliability on the learning outcomes. ${ }^{38}$ Reflecting on our exchange elective, and students' reflections in terms of bedside demonstration, case reports and integrated clinical reasoning 
learning have only two supervisors on the expert reference panel. One would propose that we will gain more learning points for $\mathrm{CR}$ if their reflections can be reviewed and discussed by more supervisors from different disciplines. Thus, we can plan our next exchange elective by adopting SCT style with involvement of more supervisors for discussion and feedback from research perspectives. Our case report writing for the students has provided a platform for case-based review of the selected cases to maximise the learning outcome. The highlighted benefit of this collaborative exchange elective is of adequate resources of cases specially for the students to learn on a preorganised teaching ward round. We need to plan multi-specialty feedback sessions at the next exchange elective. During the ensuing clinical placement, they have not only used the "SNAPPS" and "PICO" frameworks but also referred the cases to multiple supervisors for advice to increase the learning input.

Another important aspect of clinical reasoning during the elective is that recent review ${ }^{39}$ has observed clinical reasoning's variation with the clinical context influenced by patient factors, doctor factors, and environmental factors. Research about clinical reasoning has tended to focus on the individual, assessing their ability to perform clinical reasoning tasks. This review identifies areas for continued research, including which contexts have a negative or positive impact, and the effect of multiple contexts (cognitive loading) on clinical reasoning. In terms of patient factors, recent study showed 25 physicians videotaped encounters by altering one or more contextual factors including low English proficiency, emotional volatility, incorrect diagnosis suggestion, or atypical presentation. The research team found that participating physicians were more likely to misinterpret key clinical reasoning data if two contextual factors were present. The research team postulated that multiple contextual factors led to increased cognitive load, leading to a negative perception of the clinical situation, consequent mistaken interpretation, and adverse clinical reasoning outcome. ${ }^{40}$ During our exchange elective, low English proficiency may be one of the major patient factors impacting on the clinical reasoning learning despite our supervisors being shadow as language translators. Another study looked at patient's disruptive behaviour and diagnostic difficulty of presentation. Diagnostic accuracy was significantly lower for both the difficult patients $(p=0.017)$ and the diagnostically difficult cases $(p<0.001)$, however applying clinical reasoning with critical reflection did improve diagnostic rates $(p=0.002) .{ }^{41}$ The research team repeated the study by investigating why difficult patients reduced diagnostic accuracy by providing cases to 74 physicians including half with 'difficult patients' and half with 'neutral patients'. The study concluded that diagnostic scores were significantly lower for difficult patients $(p<0.01)$ and participating physicians recalled fewer clinical findings and more behaviour observations from the difficult patients $(p<0.001) .{ }^{42}$ During our elective, we have come across 'difficult patients' with behavioural issues impairing the accuracy of the clinical reasoning process and the initial clinical diagnosis. The eventual positive outcome was reached by the clinical management discussion with the patient and next of kin in a clinical priority way. The students did greatly appreciate their clinical reasoning learning through a difficult patient. Another interesting study looked at patient appearance and the effect on clinical reasoning. Participating physicians were given case-based scenarios with classed patient pictures as 'poor and dirty' in appearance or as 'rich and clean'. There was no significant difference in diagnostic accuracy, however 
participating physicians reported processing the case more extensively if the patient appeared 'rich and clean' $(p=0.04){ }^{43}$

The study has three major limitations. One was that we need to collect feedback or reflections from supervisors at the First Affiliated Hospital, Sun Yat-sen University about the students' learning during the elective. Further research should focus on feedback from both supervisors and students. A second limitation of this study is that longer term reflections and learning outcome is somewhat disrupted by the COVID-19 lockdown and online curriculum delivery. Students have very limited face-to-face access to patients and cases during the longer-term follow-up. Through further face-to-face follow-up interview with both students and supervisors, clinical reasoning will be even more integrated into their future daily clinical practice with facilitation of career choices and evidence-based care for patients. The third limitation about the study will be lack of adequate consideration of teaching clinical reasoning at real life context, especially patient factors because supervisors in charge have preselected all the cases for a teaching ward round at different disciplines.

\section{Conclusion}

These reflections provide insight into how this exchange elective may improve students' clinical reasoning skills in ongoing medical training, enhance scientific writing skills, and achieve excellent academic performance. These students accomplished their learning outcomes under joint supervision from both institutions by daily case discussion and using the clinical reasoning framework in different disciplines at the First Affiliated hospital, Sun Yat-sen University. The students have been provided many opportunities to incorporate the clinical reasoning learning into the case report writing and learning during their placements. The clinical reasoning skills learned from these experiences enhance the way clinical reasoning being taught by supervisor to improve students' clinical placement training, which has translated into their clinical academic performance and ensure the smooth transition from medical student to competent interns. This paper aims to improve the understanding of both supervisors and students in recognising the effect of this elective on motivating the ongoing learning of clinical reasoning at the bedside. The paper also suggests that short-term elective of undergraduate medical students may open an exciting avenue for further research to build upon the existing literature and innovative ways of teaching clinical reasoning.

\section{Declarations}

\section{Ethics approval and consent to participate}

\section{All study methods were carried out in accordance with relevant guidelines and regulations.}

All study protocols were approved by both the Curtin University Ethics Committee and Sun Yat-sen University Ethics Committee. Informed consents were obtained from all authors and participants at both Curtin Medical School, Curtin University, Perth, Australia and the First Affiliated Hospital, Sun Yat-Sen University, Guangzhou, China. 


\section{Consent for publication}

Consents for publication have been obtained from all authors and participants at both Curtin Medical School, Curtin University, Perth, Australia and the First Affiliated Hospital, Sun Yat-Sen University, Guangzhou, China.

\section{Availability of data and materials}

The datasets analysed during the current study are available from the corresponding author on reasonable request.

\section{Competing interests}

There are no competing interests to be declared.

\section{Funding}

There is no external funding for the study to be declared.

\section{Authors' contributions}

Dan Xu: Substantial contributions to the design of the work; the acquisition, analysis and interpretation of data for the work; Drafting the work, reviewing and revising the work critically for important intellectual content; Final approval of the version to be published; Agreement to be accountable for all aspects of the work in ensuring that questions related to the accuracy and integrity of any part of the work are appropriately investigated and resolved

Timothy Yap, Christopher Chi, Nicole Tan, Ankith Nair, Dennis Nixon, Cleo Wee: Substantial contributions to the acquisition, analysis and interpretation of data for the work; drafting the initial work, reviewing and revising the work critically for important intellectual content; Final approval of the version to be published; Agreement to be accountable for all aspects of the work in ensuring that questions related to the accuracy and integrity of any part of the work are appropriately investigated and resolved Shaoting Feng, Suqing Yang, Lucy Shuqing Ding, Johan Rosman, Sally Sandover, Jingsong Wang, Ming Kuang \& Haipeng Xiao: Contributions to the design of the work; Reviewing and revising the work critically for important intellectual content; Final approval of the version to be published; Agreement to be accountable for all aspects of the work in ensuring that questions related to the accuracy and integrity of any part of the work are appropriately investigated and resolved

\section{Acknowledgements}

We acknowledge the staff at both Curtin Medical School, Curtin University, Perth, Australia and the First Affiliated Hospital, Sun Yat-Sen University, Guangzhou, China.

\section{References}


1. Xu D, Atkinson M, Yap T, Yap M, Hossain R, Chong F, Gupta S, Hou L, Ding S, Yang Q, et al. 2020. Reflecting on exchange students' learning: Structure, Objectives and Supervision. Medical Teacher.42 (3):278-284.

2. DiCicco-Bloom B, Crabtree BF. 2006. The qualitative research interview. Medical education. 40(4):314-321.

3. Wolpaw TM, Wolpaw DR, Papp KK. 2003. SNAPPS: a learner-centered model for outpatient education. Academic Medicine. 78(9):893-898.

4. Thomas J, Harden A. 2008. Methods for the thematic synthesis of qualitative research in systematic reviews. BMC medical research methodology. 8(1):45.

5. Ziebland S, McPherson A. 2006. Making sense of qualitative data analysis: an introduction with illustrations from DIPEx (personal experiences of health and illness). Medical education. 40(5):405414.

6. Celenza A, Rogers I. 2006. Qualitative evaluation of a formal bedside clinical teaching programme in an emergency department. Emergency medicine journal. 23(10):769-773.

7. Singley MK, Anderson JR. 1989. The transfer of cognitive skill. Cambridge (MA): Harvard University Press

8. Kneebone R. 2005. Evaluating clinical simulations for learning procedural skills: a theory-based approach. Academic medicine. 80(6):549-553.

9. Sadideen H, Kneebone R. 2012. Practical skills teaching in contemporary surgical education: how can educational theory be applied to promote effective learning?. The American Journal of Surgery. 204(3):396-401.

10. Pierson DJ. 2009. How to read a case report (or teaching case of the month). Respiratory care. 54(10):1372-1378.

11. Nissen T, Wynn R. 2014. The clinical case report: a review of its merits and limitations. BMC research notes. 7(1):264.

12. Ehara S. 2011. Assessing the scientific and educational value of case reports: an editor's view. Jpn J Radiol. 29, 1-2.

13. Sun Z. 2013. Tips for writing a case report for the novice author. Journal of Medical Radiation Sciences. 60(3):108-113.

14. Har-EL G. 1999. Does it Take a Village to Write a Case Report? Otolaryngology-Head and Neck Surgery. 120(6):787-788.

15. Mishra S. 2015. Are medical breakthroughs declining-The importance of case reports?. Indian Heart Journal. 67(3):S1-S3.

16. Florek AG, Dellavalle RP. 2016. Case reports in medical education: a platform for training medical students, residents, and fellows in scientific writing and critical thinking. J Med Case Reports. 10(86).

17. Packer CD, Katz RB, lacopetti CL, Krimmel JD, Singh MK. 2017. A Case Suspended in Time: The Educational Value of Case Reports. Academic Medicine. 92(2):152-156. 
18. Schultes M,Aijaz M, Klug J,-Fixsen DL. 2021 Competences for implementation science: what trainees need to learn and where they learn it. Advances in Health Sciences Education (2021) 26:1935 https://doi.org/10.1007/s10459-020-09969-8

19. Higgs J, Jones MA, Loftus S, Christensen N, 2008. Clinical reasoning in eh health professions E-book. 3rd Ed. Philadelphia (PA): Elsevier Health Sciences.

20. Eva KW. 2005. What every teacher needs to know about clinical reasoning. Medical education. 39(1):98-106.

21. Linn A, Khaw C, Kildea H, Tonkin A. 2012. Clinical reasoning: A guide to improving teaching and practice. Australian family physician. 41(1/2):18.

22. Stanovich KE, West RF. 2000. Individual differences in reasoning: Implications for the rationality debate?. Behavioral and brain sciences. 23(5):645-665.

23. Evans JSB. 2003. In two minds: dual-process accounts of reasoning. Trends in cognitive sciences. 7(10):454-459.

24. Kahneman D. 2003. A perspective on judgment and choice: mapping bounded rationality. American psychologist. 58(9):697.

25. Croskerry P. 2009. A universal model of diagnostic reasoning. Academic medicine. 84(8):1022-1028.

26. Norman G. 2009. Dual processing and diagnostic errors. Advances in Health Sciences Education. 14(1):37-49.

27. Croskerry P. 2003. The importance of cognitive errors in diagnosis and strategies to minimize them. Academic medicine. 78(8):775-780.

28. Kahneman D. 2011. Thinking, fast and slow. New York (NY): Macmillan.

29. Pelaccia T, Tardif J, Triby E, Charlin B. 2011. An analysis of clinical reasoning through a recent and comprehensive approach: the dual-process theory. Medical education online. 16(1):5890.

30. Croskerry P, Singhal G, Mamede S. 2013. Cognitive debiasing 1: origins of bias and theory of debiasing. BMJ quality \& safety. 22(Suppl 2):ii58-ii64.

31. Schmidt HG, Mamede S. 2015. How to improve the teaching of clinical reasoning: a narrative review and a proposal. Medical education. 49(10):961-973.

32. Charlin B, Lubarsky S, Millette B, Crevier F, Audétat M, Charbonneau A, Caire Fon N, Hoff L, Bourdy C. 2012. Clinical reasoning processes: unravelling complexity through graphical representation. Medical education. 46(5):454-463.

33. Pinnock R, Welch P. 2014. Learning clinical reasoning. Journal of Paediatrics and Child Health. 50(4):253-257.

34. Braude HD. 2012. Intuition in medicine: A philosophical defense of clinical reasoning. Chicago (IL): University of Chicago Press.

35. Charlin B, Roy L, Brailovsky C, Goulet F, van der Vleuten C. 2000. The Script Concordance test: a tool to assess the reflective clinician. Teaching and learning in medicine. 12(4):189-195. 
36. Charlin B, Tardif J, Boshuizen HP. 2000. Scripts and medical diagnostic knowledge: theory and applications for clinical reasoning instruction and research. Academic medicine. 75(2):182-190.

37. Ahmadi SF, Khoshkish S, Soltani-Arabshahi K, Hafezi-Moghadam P, Zahmatkesh G, Heidari P, BabaBeigloo D, Baradaran HR, Lotfipour S. 2014. Challenging script concordance test reference standard by evidence: do judgments by emergency medicine consultants agree with likelihood ratios?. International journal of emergency medicine. 7(1):34.

38. Gagnon R, Charlin B, Coletti M, Sauvé E, Van der Vleuten C. 2005. Assessment in the context of uncertainty: how many members are needed on the panel of reference of a script concordance test? Medical education. 39(3):284-291.

39. Mason A, Locke R, Lusznat RM, Coles C, Masding MG. 2020. How Do Contexts Affect Physicians' Clinical Reasoning? A Narrative Review. MedEdPublish. 9(1):32

40. Durning S, Artino Jr AR, Pangaro L, van der Vleuten CP, Schuwirth L. 2011. Context and clinical reasoning: understanding the perspective of the expert's voice. Medical education. 45(9):927-938.

41. Schmidt HG, Van Gog T, Schuit SC, Van den Berge K, Van Daele PL, Bueving H, Van der Zee T, Van den Broek WW, Van Saase JL, Mamede S. 2017. Do patients' disruptive behaviours influence the accuracy of a doctor's diagnosis? A randomised experiment. BMJ quality \& safety. 26(1):19-23.

42. Mamede S, Van Gog T, Schuit SC, Van den Berge K, Van Daele PL, Bueving H, Van der Zee T, Van den Broek WW, Van Saase JL. Schmidt HG. 2017. Why patients' disruptive behaviours impair diagnostic reasoning: a randomised experiment. BMJ Quality \& Safety. 26(1):13-18.

43. Mohamed F, Mamede S, Mohamedani M, Al Alwan I, Magzoub MEM, Schmidt HG. 2016. The Effect of Patients $\square$ Appearance on Doctors' Diagnostic Decision Making: Do Poor People Get Poorer Medical Care?. Health Professions Education. 2(1):18-23.

\section{Tables}

Table 1 illustrated the anticipated learning opportunities and case selection and academic schedules. 


\section{Academic Schedules}

Day 1 Department of Laboratory Medicine and Department of Cardiology

Anticipated Learning Opportunities and Case Selection

Brief Introductory Lectures on haematology, serology, microbiology and immunology with case studies; Common cases (Ischaemic Heart Diseases, Heart Failure \& Atrial Fibrillation) teaching ward round, Case selection discussion

Day 2 Department of General Surgery (Thyroid and Breast) and Department of Gastrointestinal Surgery

Day 3 Department of Medical Imaging (Radiology and Nuclear Medicine) and Department of Intensive Care Unit

Day 4 Department of Hepato-Biliary Surgery and Department of Respiratory Medicine

Day 5 Department of Gastrointestinal Medicine (Endoscopy Unit) and Department of Obstetrics

Day 6 Department of Neurology and Department of Rehabilitation Medicine

Day 7 Department of Anaesthesia and Pain Medicine

Day 8 Department of Plastic Surgery and Microsurgery and Department of Vascular Surgery

Day 9 Department of Haematology and Department of Bone Marrow Transplant \& Plasmapheresis

Day 10 Department of Endocrinology and Department of Dermatology

Day 11 Department of Paediatric Medicine and
Common cases (Thyroid and Breast surgery) Teaching ward round with Case selection discussion \& Operating Rooms observation, Common cases (Upper and Lower GI malignancy and Inflammatory Bowel Diseases) Teaching ward round with Case selection discussion \& Operating Rooms observation

Brief Introductory Lectures on X-ray, Ultrasound, CT scan \& MRI Scan; Xray case studies and learn to interpret CXR, AXR and Musculoskeletal Xray with Case selection discussion; Common cases (Respiratory Failure, Multiorgan Failure and Septic shock) Teaching ward round with Case selection discussion

Common cases (Heart, Respiratory and Multi-organ failure; Renal failure and other Chronic Renal Diseases) teaching ward round, Routine Renal Medicine ward round, Physical Signs Learning and Discussion of Diagnostic workup

Lectures on basic blood test results (FBC, U+E, LFT, TFT, Fasting Glucose, $\mathrm{HbA1c}$, Lipid profile) interpretation and case studies; Histology sample preparation (Frozen section)

Lectures on X-ray, Ultrasound, CT scan and MRI Scan; X-ray case studies and learn to interpret CXR, AXR and Musculoskeletal X-ray

Common cases (Lower limb trauma, Joint replacement and Osteoarthritis) teaching ward round, Operating Rooms observation, Physical Signs Learning and Discussion of Surgical Complications; Emergency case management

Common cases (Stroke, Movement Disorder and Degenerative disorders) teaching ward round, Physical Signs Learning and Discussion of Diagnostic workup

Common cases (Ischaemic Heart Diseases, Heart Failure and Atrial Fibrillation) teaching ward round, Physical Signs Learning and Discussion of Diagnostic workup

Common cases (Type I and II Diabetes and its complications, Thyroid diseases and Pituitary diseases) teaching ward round, Physical Signs Learning and Discussion of Diagnostic workup

Common cases (Rheumatoid Arthritis, Cutaneous and Systemic Lupus and Mixed Connective tissue diseases) teaching ward round, Physical 
Department of Neonatal Intensive Care Unit

Day 12 Department of Ophthalmology and Sun Yat-Sen University Eye Hospital

Day 13 Department of Renal Medicine and Sun Yat-Sen University

Dialysis Centre

Day 14 Department of Rheumatology and Immunology and Department of Medical Oncology and Radiation Oncology
Signs Learning and Discussion of Diagnostic workup

Common cases (Asthma, Chronic Obstructive Pulmonary Diseases, Interstitial Lung Diseases and Lung Cancer) teaching ward round, Physical Signs Learning and Discussion of Diagnostic workup

Common cases (Anaemia, Clotting Disorder and Malignancy) teaching ward round, Physical Signs Learning and Discussion of Diagnostic workup

Common cases (Connective tissue diseases, Leukaemia and Lymphoma) teaching ward round, Physical Signs Learning and Discussion of Diagnostic workup

Table 2 Modified SNAPPS and PICO

SNAPPS for the exchanged students' case report writing

1. Summarize briefly the history and examination problem lists

2. Narrow the problem lists to the two commonest possibilities and one "not-to-be-missed" red-flag possibility

3. Analyse the problem lists by comparing and contrasting the possibilities

4. Probe the supervisor by asking questions about uncertainties, difficulties, or alternative approaches

5. Pick the unique case and plan for the case writing for journal submission

6. Select learning points for both case reporting and ongoing self-directed learning in the topic PICO for supervisors' \& exchanged students' bedside teaching of clinical reasoning

1. Problem lists creation in history taking, physical examination and bedside investigation

2. Investigate for supporting evidence to integrate into clinical assessment

3. Correct mistakes, teach general rules and reinforce what was right

4. Outcome analysis to identify the learning points 